\title{
Reliable Geographical Multicast Routing in Vehicular Ad-Hoc Networks
}

\author{
Maria Kihl ${ }^{1}$, Mihail Sichitiu ${ }^{2}$, Ted Ekeroth $^{1}$, and Michael Rozenberg ${ }^{1}$ \\ ${ }^{1}$ Dep. of Communication Systems, Lund University, Sweden \\ ${ }^{2}$ Dep. of Electrical and Computer Engineering, North Carolina State University, USA
}

\begin{abstract}
Vehicular ad-hoc networks (VANETs) offer a large number of new potential applications without relying on significant infrastructure. Many of these applications benefit from multi-hop relaying of information, thus requiring a routing protocol. Characteristics unique to VANETs (such as high mobility and the need for geographical addressing) make many conventional ad hoc routing protocols unsuitable. Also, some envisioned applications have end-toend QoS requirements. In this paper we propose a new multicast routing protocol specifically designed for VANETs. Its purpose is to provide a routing service for a future reliable transport protocol. We evaluate its performance using realistic network and traffic models. It is shown that it is possible to implement a reliable multicast routing protocol for VANETs.
\end{abstract}

\section{Introduction}

For many years research projects have been focused on issues regarding inter-vehicle communication (IVC) systems [1][2][3]. The objective of those projects has been to create the "fully connected vehicle". By letting vehicles communicate both with each other and with base stations along the road, accidents can be avoided and traffic information can be made available to the driver. Of course, ultimately, the vision is to have in-vehicle Internet access as well. A couple of years ago the term VANET (Vehicular Ad-hoc Network) was introduced, combining mobile ad-hoc networks (MANETs) and IVC systems.

Vehicular Ad-hoc Networks (VANETs) are envisioned to both decrease the number of deaths in traffic and improving the travel comfort by, for example, increasing intervehicle coordination. Understandably, the most commonly considered applications are related to public safety and traffic coordination. Collision warning systems and vehicle platooning are two applications that projects work on. Also, traffic management applications, traveller information support and various comfort applications have the potential to make travel (considerably) more efficient, convenient and pleasant.

Most VANET applications require that data is transmitted in a multi-hop fashion, thus prompting the need for a routing protocol. In many aspects, a VANET can be regarded as a MANET. However, the inherent nature of a VANET imposes the following three constraints for a routing protocol:

1. Short-lived links.

2. Lack of global network configuration.

3. Lack of knowledge about a node's neighbors. 
The first issue is due to the mobility of the vehicles. Studies have shown that the lifetime of a link between two nodes in a VANET is in the range of seconds [4]. Similar to a MANET, no central coordinator can be assumed in a VANET. Finally, although a hello protocol (as in OSPF) can be used to discover the neighbors of a node, this may be an expensive and difficult to tune solution. The routing protocol should discover the neighbors as needed. It is also preferable that the routing protocol works for a wide range of applications and traffic scenarios. Several papers propose solutions for specific VANET applications [5][6][7]. Some VANET applications require unicast routing. For example, some envisioned comfort applications, as on-board games and file transfer, will likely need unicast routing with fixed addresses. Many papers have proposed unicast protocols for VANETs. Some papers suggest that VANETs should use already existing unicast protocols for MANETs, as AODV [8][9] or cluster-based protocols [10][11]. Other papers propose new unicast protocols for VANETs [12][13]. However, many VANET applications require position-based multicasting (e.g., for disseminating traffic information to vehicles approaching the current position of the source). A natural match for this type of routing are the geocasting protocols

[6][14] that forward messages to all nodes within a Zone of Relevance (ZOR). The geocast concept has been studied for VANETs since the beginning of 1990s [15]. In [16] a geocasting protocol for VANETs was described; in this approach a node forwards a message after a delay that depends on the distance from the last sender. Variants of this protocol have been proposed in [17][18].

The major problem with flooding-based geocasting protocols is that the flooding mechanism is commonly based on broadcast, and it is, thus, best effort. However, some applications will require multicast transmission with end-to-end QoS. Floodingbased geocast protocols are not intended for these types of applications. Therefore, there is a need to develop multicast protocols for VANETs that can support end-toend QoS mechanisms implemented in a transport layer protocol.

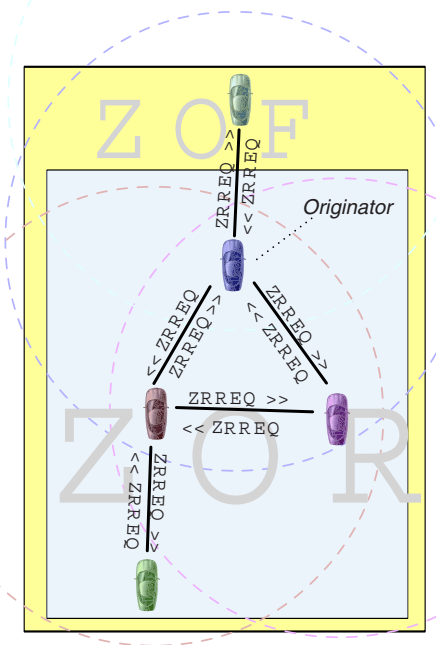

Fig. 1. ZRREQ messages are flooded from the originator (source) vehicle 
In this paper we present a RObust VEhicular Routing (ROVER) protocol, that offers reliable geographical multicast. The protocol uses a reactive route discovery process within a ZOR. We evaluate the protocol with a realistic simulation setup. We consider a generic data transfer application, in which a vehicle sends a data message to all vehicles within a specified ZOR. The results show that ROVER delivers the data with reasonable delays to $100 \%$ of the intended vehicles for almost all scenarios. Also, ROVER could be used by applications that require end-to-end QoS, by implementing a transport layer protocol that uses the multicast tree set up by ROVER.

\section{Rover}

In this section we will describe the routing protocol ROVER (RObust VEhicular Routing). In short the main difference between geocasting and ROVER is similar to the difference between flooding and a MANET reactive protocol such as AODV: both in ROVER and in AODV only control packets are flooded in the network - the data packets are unicasted, potentially increasing the efficiency and reliability. Each vehicle is assumed to have a unique Vehicle Identification Number (VIN). Also, the vehicles are assumed to have a GPS receiver and access to a digital map. The objective of the protocol is to transmit a message, $M$, from an application, $A$, to all other vehicles within an application-specified ZOR, $Z$. The ZOR is defined as a rectangle (although other definitions can be easily accommodated) specified by its corner coordinates. Thus, a message is defined by the triplet $[A, M, Z]$. When a vehicle receives a message, it accepts the message if, at the time of the reception, it is within the ZOR. Similar to geocasting protocols we also define a Zone Of Forwarding (ZOF) as a zone including the source and the ZOR. All vehicles in the ZOF are part of the routing process, although only vehicles in the ZOR deliver the message to their corresponding application layer (specified by $A$ ).

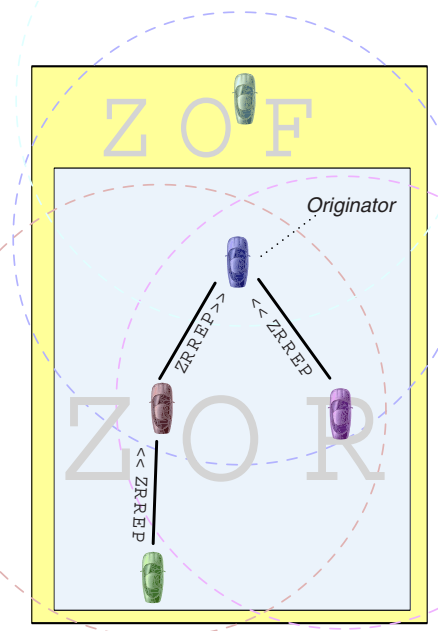

Fig. 2. ZRREP messages are unicasted the one-hop neighbors from where the ZRREQ was first received 


\subsection{Route Discovery}

The first time the routing layer receives a packet $[\mathrm{A}, \mathrm{M}, \mathrm{Z}]$ from the application layer, a route discovery process is triggered. The process is also initiated if the previous ZOR is no longer valid. The objective of the route discovery process is to build a multicast tree from the source vehicle to all vehicles within the ZOR Z.

As shown in Figure 1, the route discovery process is initiated when the originator vehicle floods a Zone Route Request (ZRREQ) message containing its VIN, location, the current ZOR, and a route sequence number, $S S$, throughout the ZOF.

Any vehicle that receives a ZRREQ for the first time for this session sequence number accepts the message if the vehicle is within the ZOF, and is not too far away from the sender. The reason for including the distance to the sender is to build a robust multicast tree. The Cutoff Distance is calculated as $\alpha \cdot R$ where $R$ is the (assumed) maximum radio range and $0<\alpha \leq 1$. In this paper we have used $\alpha=2 / 3$.

If a vehicle accepts a ZRREQ, it replies to the one-hop vehicle that forwarded the ZRREQ with a Zone Route Reply (ZRREP) message, containing its VIN. It also stores the information $[S S, Z]$ in a routing table. Finally it re-broadcasts the ZRREQ, including the original VIN, ZOR, and SS. The vehicles in ZOF but not in ZOR do not reply to ZRREQ messages unless they receive a reply themselves. The sequence number $S S$ in conjunction with the VIN of the source vehicle (originator) is used as a unique identifier in the routing tables formed by the route discovery process.

After flooding the ZRREQ throughout the ZOF, unlike for AODV, the ZRREP messages are not sent back to the source. Instead they are only transmitted to the node transmitting the ZRREQ. All recipients of a ZRREP message store the VIN of the vehicle that sent the ZRREP and the corresponding SS and source VIN. Data packets from the same source VIN and SS will be forwarded to the sender of the ZRREP. This way all nodes store the local information needed to build a multicast tree rooted at the source node. Once the tree is formed, i.e., after the ZRREP are sent to parents in the tree, data can be disseminated in the tree (as shown in Figure 2).

\subsection{Data Transfer}

Since each vehicle stores next-hop(s) information about the source VIN and SS, data will be forwarded through the tree as a function of those numbers. The source forwards the data packets immediately after it receives a ZRREP message. The source (and all forwarding nodes in the multicast tree) unicasts the message $M$ to all the vehicles from which it received a ZRREP. The message is also stored in a buffer for a short time in case it receives a ZRREP after it receives the message. Thus, each message is propagated through the multicast tree according to the "route table" stored during the route discovery process. All receivers also deliver the packet if they are within the ZOR. Since the data is transferred using unicast, it benefits of the normal MAC-layer acknowledgments.

\subsection{Route Timeout}

As vehicles move, the ZOR for a certain application will change in time. However, if a vehicle sends several messages to the same ZOR application within a short time, 
there is no need to perform a route discovery for each message. For example, for vehicles travelling at $90 \mathrm{~km} / \mathrm{h}$, the ZOR may only change by $25 \mathrm{~m}$ in one second. If the initial ZOR is several kilometers large, the same ZOR can be used. We considered a ZOR invalid when the source vehicle moved for more than $25 \mathrm{~m}$ from the initial route discovery position.

\section{Simulation Environment}

We have evaluated ROVER using the simulation package Jist/SWANS [19][20] with the STRAW module [21]. Jist/SWANS is a simulator for mobile ad-hoc networks, similar to ns-2, implemented in JAVA. STRAW uses real maps from the Topologically Integrated Geographic Encoding and Referencing (TIGER) system available from the US Census Bureau Geography [22]. We enhanced the simulation setup in several respects and implemented ROVER as a new routing module. At the time we performed the simulations, the development of Jist/SWANS is an ongoing project, and the STRAW module is developed for city scenarios with low speeds and a road grid. It also had a number of incomplete protocol specifications (e.g., missing sequence number in 802.11). Furthermore, since the original protocol stack uses unicast with fixed addresses, we had to make a number of modifications to the original Jist/SWANS/STRAW packages.

\subsection{A Data Transfer Application}

To evaluate the performance of the proposed routing protocol, we used a generic data transfer application. In this application a vehicle sends a message to vehicles behind it. The vehicle that sends out the message will be referred to, in the rest of the paper, as the Source Vehicle (SV). When an SV sends a message, the application determines a suitable ZOR. In this paper, the ZOR will be a rectangle directly behind the SV, with length $L$ meters and width $W$ meters. $W$ is large enough to cover all lanes of the current road that goes in the same direction as the SV. The message should then be delivered to all vehicles within the ZOR, as fast and as reliable as possible.

\subsection{Road and Traffic Models}

STRAW uses real road maps by default. Since the objective of the investigations was to evaluate the proposed routing protocol, we wanted to have a very simple road model to avoid any effects caused by the specific road map used. Therefore, we constructed a straight highway in TIGER format and then used this road in the simulations. The highway is of length $10 \mathrm{~km}$ and with 3 lanes in each direction. The maximum allowed speed on the highway is 120 kilometers per hour.

Vehicles move according to a car-following model [23]. We implemented lane changing behavior. Originally STRAW did not implement this feature, and we observed cases with one lane was heavily congested while the other one was not. In our setup vehicles may change lane if the vehicle in front of them moves too slow. 


\subsection{Communication Models}

At the physical layer we used the Rayleigh fading model supplied by SWANS. This model has a gradual transition from $100 \%$ to $0 \%$ reception rate as the distance between the sender and receiver increases. The physical layer data rate we considered was $54 \mathrm{Mbps}$, consistent with the 802.11a data rates (which in turn are similar to the Dedicated Short Range Communications (DSRC) standard [24]).

At the MAC layer we used the CSMA/CA scheme used in IEEE 802.11 (similar to DSRC). At the network and transport layer we used a slightly modified version of IP and UDP. In particular, since we used geographical addressing, instead of the normal IP addresses we used VIN numbers for the vehicles and ZOR and ZOF (specified by the coordinates of the corners) to specify the destinations.

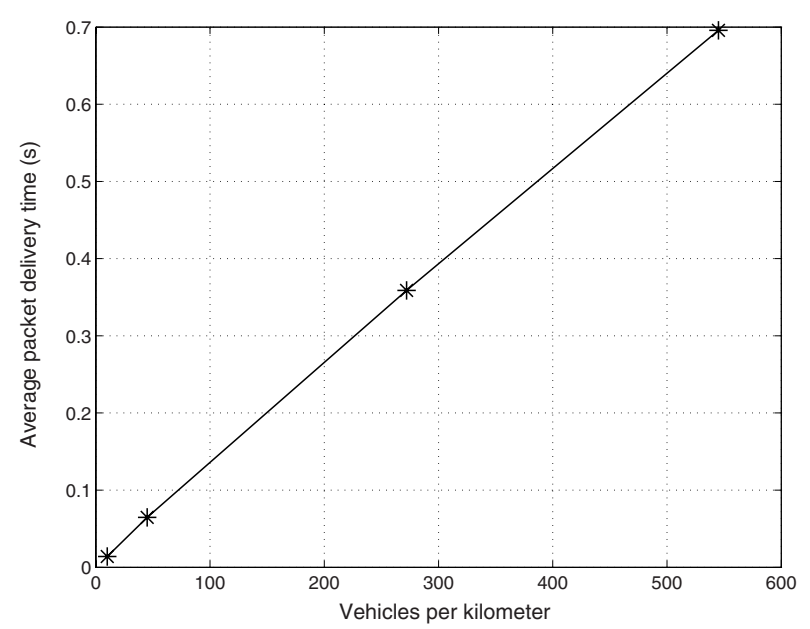

Fig. 3. Varying number of vehicles

\subsection{Simulation Setup}

In the beginning of a simulation, a number $N$ of vehicles are placed on the highway at regular intervals. All vehicles attempt to travel with the maximum posted speed while using the car following model. We placed the vehicles uniformly on the highway. Three seconds into the simulation a vehicle sends a message to vehicles in a ZOR behind itself. In our implementation the ZOR specifies the following:

- The VIN of the source node.

- The current location of the source node (absolute coordinates).

- The extent of the ZOR (relative to the source node).

- The direction of the movement of the source node.

The maximum deviation of the direction of a vehicle from the direction of the SV such that it can still be considered in the ZOR. Nodes that deviate from more than this specified value are in ZOF but not in ZOR. In our implementation we used 180 
degrees (i.e., all vehicles in the ZOR will deliver their packets to the application layer). The ZOF (for this application) is specified as the ZOR and an additional buffer zone 15 meters wide.

The default simulation parameters (shown in parenthesis) and the range of values we investigated are shown in Table 1. During the simulations we varied one parameter at a time while maintaining the rest fixed at the default value.

Table 1. Simulation parameters

\begin{tabular}{|l|l|}
\hline Parameter & Value \\
\hline Number of vehicles/km & $10,45,(272), 545$ \\
\hline Radio transmission range [m] & $100,200,(300), 400$ \\
\hline Length of the ZOR [km] & $0.5,(1.5), 2.5,3.5$ \\
\hline
\end{tabular}

\section{Performance Metrics}

We used two performance metrics when evaluating ROVER. The first metric we considered was the packet delivery ratio, $P D R$, i.e. the percentage of vehicles that (1) are within the ZOR when the message is sent and (2) receives the message. To measure the PDR for each message from the $S V$ we counted the number of vehicles in the ZOR at the time the message was generated and compared it with the number of vehicles that receive the message. Since more vehicles can enter the ZOR before the message is transmitted throughout the ZOR, PDR can be (slightly) larger than $100 \%$. The second metric was the average packet delivery time, $T_{D}$, i.e., the average delay between the time a message is sent by the $S V$ until the vehicles receives the message.

\section{Results and Discussion}

In this section we present the performance results for varying the vehicle density, the transmission range and the size of the zone of relevance. The results shown here are averages from 30 runs (with different seeds), and all confidence intervals are within $10 \%$ of the average.

\subsection{Packet Delivery Ratio (PDR)}

The results show that ROVER delivers $100 \%$ of the messages for almost all scenarios. It is only when the vehicle density is very low $(10$ vehicles $/ \mathrm{km})$ that a message sometimes cannot reach all vehicles within the ZOR. In this case, the average distance between the vehicles is 100 meters, which means that if a ZZREQ or a ZZREP message is lost, a part of the multicast tree may be lost. However, this result is not only due to ROVER, since all routing protocol would probably encounter problems for such low vehicle densities. For all other scenarios, the PDR is $100 \%$.

It can be noted that the cutoff mechanism in the route discovery process has a major impact on the performance. It is crucial that the multicast tree is robust and 


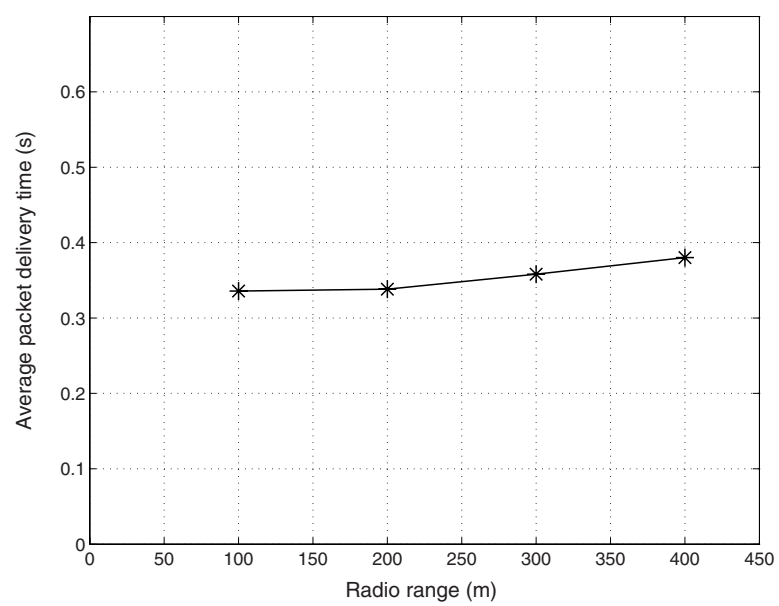

Fig. 4. Varying radio range

therefore it is important that linked nodes are relatively close to each other due to the fading channel. It is better with several short (reliable) hops than a few long (unreliable) hops.

\subsection{Packet Delivery Time $\left(\mathrm{T}_{\mathrm{D}}\right)$}

The packet delivery time, $T_{D}$, shows the average time it takes for the message to reach all cars within the ZOR. Of course, the results depend on the chosen scenario.

\subsubsection{Vehicle Density}

As the number of cars increases, $T_{D}$ also increases, see Figure 3 . The Route Discovery Process is based on flooding. With more cars, packet collisions and backoff times increase at the MAC layer and the effect is longer delays on the application layer. Several papers (see, for example, [16]) have suggested an improved flooding mechanism in which a node has a waiting time before forwarding a packet. The waiting time depends on the distance to the previous sender and nodes further away from the sender will forward the packet sooner than nodes close to the sender. We implemented this feature in ROVER, but could not see any obvious improvements in the performance.

\subsubsection{Radio Transmission Range}

One could expect that a longer radio range would decrease the packet delivery time, due to fewer hops. However, our results for these scenarios showed that the radio range is not a major factor in the delivery time, see Figure 4. As the transmission range increases, each transmission will be heard by more nodes. Therefore, the risk of packet collisions and hidden terminals increases. Also, one major part of the packet delivery time is the protocol handling delay in the nodes. This delay will of course not be shorter just because the radio range increases. 


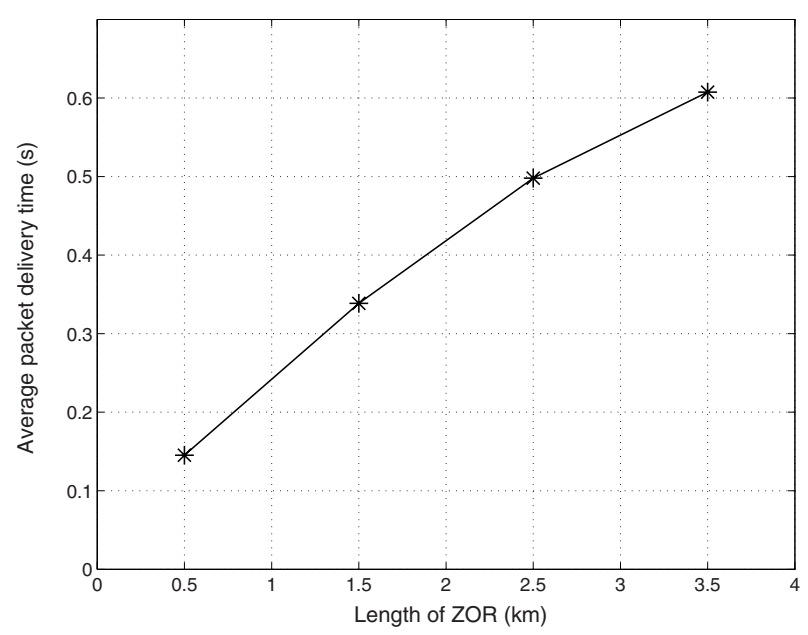

Fig. 5. Varying length of ZOR

\subsubsection{Zone of Relevance}

As expected, the delivery time is proportional to the length of the ZOR, see Figure 5 . More hops are needed to cover the larger area and therefore the delivery time increases. Remarkable is that even for a ZOR as long as $3.5 \mathrm{~km}$, the delivery time is as low as $600 \mathrm{~ms}$ and $100 \%$ of the vehicles within the ZOR receives the data. Therefore, ROVER is well suited for VANET applications that require multicast with end-to-end QoS.

\section{Conclusions}

Vehicular ad-hoc networks have the potential to both reduce accidents as well as enhance the comfort of the driver and passengers. Different applications will have different enforce different requirements on the network protocols used. In this paper we have focused on those applications that have requirements on the end-to-end QoS. For those applications there will be a need for a reliable transport protocol. In order for a reliable transport protocol to work properly, a routing protocol is needed that maintains some information about sender and receivers.

Therefore, we in this paper have presented ROVER, a new multicast routing protocol for vehicular ad hoc networks. The protocol uses geographical addressing to form a multicast tree within a zone of relevance. The tree is formed on-demand and can be used to forward multiple data packets from the same source. Therefore, it can be used by a reliable transport protocol to ensure end-to-end QoS. We have evaluated the performance of the protocol in a realistic environment with detailed models both for the vehicular traffic as well as for the physical environment. 


\section{References}

1. M. Williams, "PROMETHEUS-the european research programme for optimising the road transport system in europe", In Proc. of the IEE Colloquium on 'Driver Information' (Digest No.127), 1988, pp. 1-9.

2. S. Shladover, C. Desoer, J. Hedrick, M. Tomizuka, J. Walrand, W.Zhang, D. McMahon, H. Peng, S. Sheikholeslam, and N. McKeown, "Automatic vehicle control developments in the PATH program", IEEE Transactions on Vehicular Technology, vol. 40, no. 1, pp. 114-130, 1991.

3. W. Enkelmann, "Fleetnet - applications for inter-vehicle communication," in Proc. of the IEEE Intelligent Vehicles Symposium, 2003, pp. 162-167.

4. S. Wang, "The effects of wireless transmission range on path lifetime in vehicleformed mobile ad hoc networks on highways", In Proc. of the IEEE International Conference on Communications, 2005, pp. 3177-3181.

5. S. Biswas, R. Tatchikou, and F. Dion, "Vehicle-to-vehicle wireless communication protocols for enhancing highway traffic safety", IEEE Communications Magazine, vol. 44, pp. 74-82, 2006.

6. C. Maihöfer, C. Cseh, W. Franz, and R. Eberhardt, "Performance evaluation of stored geocast", In Proc. of IEEE 58th Vehicular Technology Conference, 2003, pp. 29012905.

7. J. Bronsted and L. Kristensen, "Specification and performance evaluation of two zone dissemination protocols for vehicular ad-hoc networks", In Proc. of the 39th Annual Simulation Symposium, 2006.

8. T. Kosch, C. Schwingenschlögl, and L. Ai, "Information dissemination in multi-hop inter-vehicle networks", In Proc. of the IEEE 5th International Conference on Intelligent Transportation Systems, 2002, pp. 685-690.

9. A. Ho, Y. Ho, and K. Hua, "A connectionless approach to mobile ad hoc networks in street environments", In Proc. of the 2005 IEEE Intelligent Vehicles Symposium, 2005, pp. 575-582.

10. R. Santos, A. Edwards, R. Edwards, and N. Seed, "Performance evaluation of routing protocols in vehicular ad-hoc networks", International Journal of Ad Hoc and Ubiquitous Computing, vol. 1, pp. 80-91, 2005.

11. T. Little and A. Agarwal, "An information propagation scheme for VANETs", In Proc. of the 8th International IEEE Conference on Intelligent Transportation Systems, 2005, pp. $155-160$.

12. C. Lochert, H. Hartenstein, J. Tian, H. Füssler, D. Hermann, and M. Mauve, "A routing strategy for vehicular ad hoc networks in city environments", in Proc. of the IEEE Intelligent Vehicles Symposium, 2003, pp. 156-161.

13. G. Korkmaz, E. Ekici, F. Özgüner, and U. Ösgüner, "Urban multi-hop broadcast protocol for inter-vehicle communication systems", In Proc. of the first ACM workshop on Vehicular ad hoc networks, 2004, pp. 76-85.

14. Y. Ko and N. Vaidya, "Flooding-based geocasting protocols for mobile ad hoc networks," Mobile Networks and Applications, no. 7, pp. 471-480, 2002.

15. W. Kremer, "Realistic simulation of a broadcast protocol for a inter vehicle communication system (IVCS)", In Proc. of the 41st IEEE Vehicular Technology Conference, 1991, pp. 624-629.

16. L. Briesemeister, L. Schäfers, and G. Hommel, "Disseminating messages among highly mobile hosts based on inter-vehicle communication", In Proc. of the IEEE Intelligent Vehicle Symposium, 2000, pp. 522-527. 
17. H. Alshaer and E. Horlait, "An optimized adaptive broadcast scheme for inter-vehicle communicatiorn”, In Proc. of the IEEE 61st Vehicular Technology Conference, 2005, pp. 2840-2844.

18. A. Bachir and A. Benslimane, "A multicast protocol in ad hoc networks inter-vehicle geocast", In Proc. of the 58th IEEE Vehicular Tech nology Conference, 2003.

19. R. Barr, Z. J. Haas, and R. van Renesse, "Jist: An efficient approach to simulation using virtual machines", Software - Practice and Experience, vol. 35, pp. 539-576, 2004.

20. SWANS website, http://jist.ece.cornell.edu.

21. D. Choffnes and F. Bustamante, "An integrated mobility and traffic model for vehicular wireless networks", In Proc. of the Sec-ond ACM International Workshop on Vehicular Ad Hoc Networks (VANET 2005), Cologne, Germany, Sep 2005.

22. US Census Bureau Geography, http://www.census.gov/geo/www.

23. R. Rothery, "Car following models", Trac Flow Theory, 1992.

24. "Standard specification for telecomunications and information exchange between roadside and vehicle systems - $5 \mathrm{GHz}$ band dedicated short range communications (DSRC) medium access control (MAC) and physical layer (PHY)," ASTM E2213-03, Sept. 2003. 